11-ARTIGO ORIGINAL

\title{
Aderência e atividade microbicida de monócitos em portadores de esquistossomose mansônica na forma hepatoesplênica cirúrgica ${ }^{1}$
}

\author{
Carlos Teixeira Brandt ${ }^{2}$ \\ Carlos Roberto Carvalho Leite ${ }^{3}$ \\ Raul Manhães-de-Castro ${ }^{4}$ \\ Carlos Brandt Filho ${ }^{5}$ \\ Célia Maria Machado Barbosa de Castro ${ }^{6}$
}

\begin{abstract}
Brandt CT, Leite CRC, Manhães-de-Castro R, Brandt Filho C, Castro CMMB. Aderência e atividade microbicida de monócitos em portadores de esquistossomsoe mansônica cirúrgica. Acta Cir Bras [serial online] 2003 Mar-Abr;18(2). Disponível em URL: http://www.scielo.br/acb.
\end{abstract}

RESUMO - A cirurgia nas crianças portadoras de esquistossomose mansônica inclui esplenectomia, ligadura da veia gástrica esquerda e o auto-implante de tecido esplênico no omento maior. A eficácia desse procedimento pode ser responsável pelo desaparecimento da sepse fulminante pósesplenectomia (SFPE) neste tipo de paciente. Esta condição é atribuída à diminuição de $\operatorname{IgM}$, de linfócitos circulantes, de properdina e ausência de tuftsina, o que conduz a deficiência da atividade das células macrófágicas, que são responsáveis pela aderência à bactéria, fagocitose e destruição das mesmas. Objetivo: Analisar os aspectos funcionais dos monócitos destes pacientes, operados quando crianças, no Serviço de Cirurgia Geral da Criança do Hospital das Clínicas da UFPE, entre 1991 a 2000. Métodos: Foram analisados os índices de aderência in vitro dos monócitos e a geração do ânion superóxido $\left(\mathrm{O}_{2}^{-}\right)$, em três grupos. $\mathrm{O}^{1}$, auto-implante (AI), constituído por 18 portadores de esquistossomose mansônica na forma hepatoesplênica, submetidos a esplenectomia, ligadura da veia gástrica esquerda e auto-implante de tecido esplênico no omento maior; o $2^{\circ}$, (ESP), formado por nove pacientes similares, submetidos a esplenectomia e desconexão ázigoportal, e o $3^{\circ},(\mathrm{CT})$, constituído por 12 adolescentes sadios, oriundos da mesma condição sócioeconômica-geográfica. Resultados: Não houve diferença no índice de aderência entre os três grupos. Os monócitos dos pacientes do grupo AI tiveram a geração de $\mathrm{O}_{2}^{-}$semelhante à dos indivíduos do grupo CT, e significantemente maior do que os pacientes do grupo ESP. Conclusões: Os monócitos dos portadores de esquistossomose hepatoesplênica submetidos a esplenectomia, ligadura da veia gástrica esquerda e auto-implante de tecido esplênico no omento maior se mostram funcionalmente similares aos de indivíduos normais da mesma condição sócio-econômica-geográfica.

DESCRITORES - Esquistossomose mansônica. Monócitos. Atividade microbicida.

1. Trabalho realizado no Serviço de Cirurgia Geral da Criança HC - UFPE e no LIKA.

2. Prof. Titular de Clínica Pediátrica Cirúrgica, CCS - UFPE.

3. Mestre em Cirurgia, CCS - UFPE.

4. Prof. Adjunto em Nutrição, CCS - UFPE.

5. Bolsista de Iniciação Científica - CNPq/UFPE.

6. Prof. Adjunto em Medicina Tropical, CCS - UFPE. 


\section{Introdução}

A esquistossomose persiste como problema médico-social no Nordeste do Brasil ${ }^{1}$. Na criança, sua forma hepatoesplênica (EHE) pode determinar hipertensão porta, sangramento digestivo, hiperesplenismo e alterações do crescimento e desenvolvimento ${ }^{2}$. A maioria desses pacientes é tratada clinicamente, mas alguns requerem intervensão cirúrgica, sendo a esplenectomia, ligadura da veia gástrica esquerda e autoimplante de tecido esplênico uma opção freqüente ${ }^{2,3}$. $\mathrm{Na}$ vigência de recidiva hemorrágica é indicada esclerose endoscópica das varizes de esôfago ${ }^{4}$.

A esplenectomia determina alterações imunitárias, em virtude da perda da função imunológica de proteção do baço $o^{2,5}$. A complicação mais temida é a sepse fulminante pós-esplenectomia (SFPE), que tem mortalidade de $50 \%$ a $75 \%$.

$\mathrm{O}$ auto-implante de tecido esplênico é uma forma aceitável de preservar a função do baço quando a esplenectomia total for inevitável ${ }^{7,8}$. O procedimento diminui a ocorrência de SFPE, que é atribuída à diminuição de: IgM, linfócitos, properdina e ausência de tuftsina, conduzindo à deficiência da atividade das células mononucleares, que são responsáveis pela fagocitose e destruição de bactérias ${ }^{9-11}$.

A aderência dos monócitos à superfície bacteriana é a primeira etapa da ativação dessas células e início da resposta imunológica ${ }^{12}$. Na seqüência, as células fagocitárias destroem as bactérias utilizando sistemas microbicidas dependente de oxigênio, constituídos de oxidantes letais ${ }^{13}$. Logo, uma alteração no balanço oxidativo celular possui papel chave em condições fisiológicas e patológicas, levando a vários estados de morbidade $^{14}$.

Diversos estímulos induzem, em fagócitos, eventos metabólicos, tais como a explosão respiratória. Neste fenômeno, o oxigênio molecular $\left(\mathrm{O}_{2}^{-}\right)$sofre uma redução enzimática para produzir agentes microbicidas. A reação chave na explosão respiratória é a redução do oxigênio a ânion superóxido $\left(\mathrm{O}_{2}^{-}\right)$. $\mathrm{O} \mathrm{O}_{2}^{-}$é fabricado pelos leucócitos através da NADPH-oxidase, essa enzima catalisa a redução de um elétron de oxigênio. $\mathrm{O}$ $\mathrm{O}_{2}{ }^{-}$gerado pode ser convertido a peróxido de hidrogênio $\left(\mathrm{H}_{2} \mathrm{O}_{2}\right)$ na presença da superóxido dismutase (SOD). Por isso, essas enzimas são importantes na proteção da célula contra a ação do ânion $\mathrm{O}_{2}^{-}$, permitindo sua ação no centro do fagossomo ${ }^{15,16}$.

$\mathrm{O} \mathrm{O}_{2}^{-}$, produzido durante a fagocitose, pode ser detectado através de sua habilidade em reduzir um aceptor de elétrons. $\mathrm{O}$ ferricitocromo $\mathrm{C}$ tem sido usado neste intuito ${ }^{17}$. Assim, modificações na análise de redução do ferricitocromo C pela SOD têm sido usadas para detectar a geração de $\mathrm{O}_{2}$ - por neutrófilos, monócitos e macrófagos ${ }^{17,18}$.

O presente estudo teve por objetivo avaliar a atividade funcional de monócitos através do índice de aderência e da geração de superóxido, tratados ou não com tuftsina, em portadores de esquistossomose mansônica na forma hepatoesplênica, submetidos a esplenectomia, ligadura da veia gástrica esquerda e autoimplante de tecido esplênico no omento maior.

\section{Métodos}

A pesquisa foi aprovada pela Comissão de Ética do Centro de Ciências da Saúde da UFPE. O termo de consentimento foi assinado por todos os pacientes ou responsáveis.

\section{Pacientes}

Foram constituídos três grupos: $\mathrm{O}$ grupo teste (auto-implantados - AI) incluiu 18 adolescentes portadores de EHE, submetidos, no período de 1991 a 2000, no Serviço de Cirurgia Geral da Criança do HC da UFPE, a esplenectomia, ligadura da veia gástrica esquerda e auto-implante de tecido esplênico no omento maior. Todos foram submetidos a avaliações clínica, laboratorial, endoscópica e ultra-sonográfica no préoperatório e a cintilografia com enxofre coloidal marcado com Tecnécio $99^{\mathrm{m}}$, no pós-operatório.

A confirmação da infecção esquistossomótica foi feita pelo Kato-Katz. Todos os pacientes haviam sido tratados com dose única de oxaminiquine $(20 \mathrm{mg} / \mathrm{Kg}$ de peso, via oral) e submetidos a tratamento cirúrgico após 30 dias. O seguimento pós-operatório variou de 1 a 10 anos, média $(5,5 \pm 2,4)$. Foram excluídos os pacientes com: antiginemia positiva para hepatite; uso abusivo de bebidas alcoólicas; biópsia hepática com lesões sugestivas de doença hepática alcoólica; uso de imunosupressores ou estimulantes do sistema imunológico durante o período do estudo; e os pacientes com doenças hematológicas. A idade variou de 14 a 23 anos, média $(18,5 \pm 2,4)$. Dez eram do sexo masculino $(55,5 \%)$ e oito do feminino $(44,5 \%)$. Os pacientes tinham, à época da cirurgia, idades entre 9 e 18 anos, média $(12,9 \pm 2,1)$.

Em relação à comprovação do auto-implante dos tecidos esplênicos, houve captação do enxofre coloidal marcado com Tecnécio ${ }^{99} 100 \%$ dos casos. Nenhuma criança teve SFPE.

O grupo controle esplenectomizado (ESP) foi constituído por nove adultos, idades de 23 a 31 anos, média $(27,4 \pm 2,8)$, portadores de EHE submetidos a 
esplenectomia e desconexão ázigo-portal. O seguimento pós-operatório variou de 1 a 6 anos, média $(2,9 \pm 1,6)$. Sete pacientes eram do sexo masculino e dois do feminino. $\mathrm{O} \mathrm{HbsAg}$ foi negativo em todos os casos.

O grupo controle sadio (CT) foi constituído por 12 adolescentes voluntários sadios, idades variando de 15 a 19 anos, média $(16,6 \pm 1,2)$, não portadores de esquistossomose, sem antecedentes de banho de rio, oriundos do município de Timbaúba -PE. Dois eram do sexo masculino $(16,6 \%)$ e 10 do feminino $(83,3 \%)$. Nenhum voluntário tinha morbidade associada. O exame físico destes adolescentes foi normal.

\section{Procedimentos}

Os pacientes foram operados sob anestesia geral. Após a esplenectomia, o baço era imerso em recipiente contendo solução de Ringer-lactato à temperatura de \pm 4 graus Celsius $\left({ }^{\circ} \mathrm{C}\right)$. $\mathrm{O}$ pólo superior era seccionado em fragmentos de aproximadamente $2 \times 2 \mathrm{~cm}$, e implantados no grande omento, sendo fixados com categute cromado 000 , e envoltos por esta estrutura. $\mathrm{O}$ peso total dos implantes era cerca de $100 \mathrm{~g}$.

O estudo foi do tipo ensaio biológico, sendo objeto de estudo in vitro, os monócitos do sangue periférico. A parte laboratorial foi realizada no Setor de Microbiologia do Laboratório de Imuno Patologia Keiso Azami (LIKA).

Foram coletados $20 \mathrm{ml}$ de sangue dos indivíduos de todos os grupos. O sangue era distribuído em quatro containers à vácuo, de $5 \mathrm{ml}$ cada, e contendo EDTA a $15 \%$.

O experimento seguiu o protocolo descrito por Johnston ${ }^{17}$. O sangue era distribuído em tubos para centrífuga. Posteriomente, diluído na proporção de 1:2 em meio de cultura Roswell Park Memorial Institute (RPMI 1640), estéril a $8^{\circ} \mathrm{C}$, e, aos $40 \mathrm{ml}$ da suspensão eram adicionados $10 \mathrm{ml}$ de Histopaque (SIGMA). A mistura era centrifugada por 30 minutos a $1.400 \mathrm{rpm}$ $\left(2^{\circ}\right.$ a $\left.8^{\circ} \mathrm{C}\right)$. A seguir, era coletado, com pipeta de Pauster, a camada formada por mononucleares (PBMC), que era transferida para outro tubo de centrífuga. Eram adicionados $15 \mathrm{ml}$ de meio RPMI 1.640 , e feito, em seguida, nova centrifugação por 10 minutos. O sobrenadante era aspirado e desprezado. Posteriormente, o sedimento era ressuspendido em $2 \mathrm{ml}$ de meio de cultura RPMI 1640, contendo soro fetal bovino a $3 \%$ e antibióticos (penicilina $100 \mathrm{U} / \mathrm{ml}$ e estreptomicina $100 \mathrm{mg} / \mathrm{ml}$ ). Desta última solução, era retirada alíquota de $10 \mathrm{ml}$ e colocada em tubo Eppendorf, plástico e estéril, e adicionado, à suspensão de células, $90 \mathrm{ml}$ do corante azul tripan a $0,05 \%$. Este corante, portanto, foi utilizado para fazer a contagem das células e avaliar a sua viabilidade. Do total de células recuperadas, os monócitos encontravam-se em uma proporção de $95 \%$. Ao final, a mistura era disposta sobre uma lâmina espessa de vidro (Hemocitômetro ou Câmara de Neubauer), que era levada ao microscópio óptico para contagem das células.

O resultado da contagem das células era obtido aplicando-se a seguinte fórmula: Células $/ \mathrm{mm}^{3}$ de sangue $=\mathrm{n} \times 10 \times 10 \times 10^{3}$ onde: $\mathrm{n}$ : número total de células contadas nos quatro campos; 10: fator de conversão da diluição; 10: fator da espessura lamínula/lâmina; $10^{3}$ : fator de conversão para $1 \mathrm{~mm}^{3 ;} \mathrm{A}$ concentração celular foi ajustada para $1.10^{6}$ células / $\mathrm{ml}$.

Posteriormente, eram adicionados $6 \mathrm{ml}$ de meio RPMI 1.640 aos $2 \mathrm{ml}$ de células já existentes. As células eram colocadas em placas de cultura contendo seis poços de $35 \mathrm{~mm}$ de diâmetro cada, onde eram dispensados $2 \mathrm{ml}$ da suspensão de células em uma proporção de $10^{6}$ células $/ \mathrm{ml}$ de meio de cultura RPMI 1.640 , em cada poço. O material era colocado na incubadora a $37^{\circ} \mathrm{C}$ e $5 \%$ de dióxido de carbono $\left(\mathrm{CO}_{2}\right)$ Após, era feita lavagem do poço com meio de cultura RPMI 1.640 para retirada das células não aderentes à superfície da placa. Posteriormente, a placa era deixada por mais $1 \mathrm{~h}$ na incubadora para a preparação do ensaio da geração de radical superóxido.

Após a recuperação dos monócitos, as células eram ressuspendidas em RPMI 1.640. Alíquota de $10 \mathrm{ml}$ da suspensão de células não aderentes era colocada em tubo Eppendorf, misturadas a $90 \mathrm{ml}$ do corante azul tripan a $0,05 \%$ e contadas.

O IA era calculado usando a fórmula ${ }^{19}$. $\mathrm{IA}=100-\left(\underline{\mathrm{n}^{\mathrm{o}} \text { de macrófagos não aderidos } / \mathrm{ml}} \times 100\right)$ $\mathrm{n}^{\mathrm{o}}$ inicial de macrófagos

Os monócitos aderidos eram utilizados para determinar a geração de superóxido, cuja liberação era induzida com PMA (SIGMA) em solução salina balanceada de Hank's (HBSS, Gibco), concentração de $2 \mathrm{ml} / \mathrm{ml}$. Foi utilizado o ferricitocromo $\mathrm{C}$ de mitocôndria de coração de cavalo, tipo IV $(30 \mathrm{mg} / \mathrm{ml}$ em HBSS, $2.4 \times 10^{-3}$ M, Sigma Chemical Company, St. Louis, MO, USA). A especificidade da geração de superóxido foi conseguida pela utilização da superóxido dismutase de eritrócitos bovinos - SOD (SIGMA), contendo $3.000 \mathrm{u} / \mathrm{mg}$ de proteína em solução final de $3 \mathrm{mg} / \mathrm{ml}$.

Para a avaliação da produção de superóxido foram preparados três sistemas de análise descontínua com avaliação a cada uma hora, durante um período de duas horas. Para o preparo destes sistemas, foram utilizados os monócitos do sangue em cultura $(2 \mathrm{ml} /$ poço com 
$10^{6} / \mathrm{ml}$ de meio de cultura). Estes sistemas eram mantidos em incubadora com atmosfera úmida a 5\% de $\mathrm{CO}_{2}$ a $37^{\circ} \mathrm{C}$, por 10 minutos, para ativação da SOD.

No primeiro sistema em que foi adicionado SOD, (utilizada para inibir a produção de radical superóxido formado e garantir a especificidade do experimento), $\mathrm{o}$ meio foi acrescido de citocromo C e PMA (acetato miristato de forbol - PMA, SIGMA) preparado em solução concentrada de $3000 \mathrm{mg} / \mathrm{ml}$ em dimetil sulfóxido de sódio (DMSO, SIGMA). O PMA utilizado para estimular as células era diluído para uma concentração de $2 \mathrm{mg} / \mathrm{ml}$ em $2.145 \mathrm{ml}$ de solução salina balanceada de Hanks (HBSS, GIBCO) e colocado nos poços da placa de cultura. No segundo sistema, contendo água destilada, também foram adicionados citocromo $\mathrm{C}$ e PMA. O terceiro sistema continha Citocromo C, PMA e Tuftsina, esta última usada para avaliar a sua influência na geração de radical superóxido.

Em seguida, amostras de $600 \mathrm{ml}$ eram retiradas concomitantemente de cada sistema e colocadas em tubos Eppendorf. A primeira alíquota recolhida relativa ao tempo "zero" de cada sistema correspondia ao branco do espectrofotômetro. Em seguida, a reação era paralisada em banho de gelo. As amostras subseqüentes eram coletadas em intervalos de tempo regulares, nas mesmas condições, de uma em uma hora, até duas horas.

Ao término da última coleta, as amostras eram centrifugadas a $10.000 \mathrm{rpm}$ por $5 \mathrm{~min}$ à temperatura ambiente em microcentrífuga $(25.000 \mathrm{Xg}$ - rotor Ra$1 \mathrm{M}$ Kubota). Ao final da coleta, as células aderidas na placa eram recuperadas em cloreto de sódio a $0,9 \%$ para determinar o conteúdo em proteínas. As amostras eram levadas para determinação do grau de redução de ferrocitocromo $\mathrm{C}$ usando-se cubeta de $1 \mathrm{ml}$ (quartzo) e espectrofotômetro com comprimento de onda ajustado para a faixa do vermelho $(550 \mathrm{~nm})$. Os resultados da leitura eram registrados como valores de DO ou absorbância.

Sabendo-se que $1 \mathrm{~mol}$ de superóxido reduz $1 \mathrm{~mol}$ de citocromo $\mathrm{C}$, para converter os resultados obtidos na leitura do espectrofotômetro (DO) para nmoles de $\mathrm{O}_{2}^{-} / \mathrm{min}$, foi confeccionado uma curva correlacionando os resultados das leituras do espectro com os respectivos tempos de coleta, e nesta curva encontrado o valor correspondente em DO, ao tempo 1 minuto. Em seguida, multiplicava-se o valor encontrado pela constante 71,4 , quando o volume total do sistema era de $1,5 \mathrm{ml}$. Esta constante representava o valor em DO obtido a $550 \mathrm{~nm}$ para um sistema de $1,5 \mathrm{ml}$ de citocromo $\mathrm{C}$, em uma cubeta de $\mathrm{cm}^{2}$, onde todo o citocromo $\mathrm{C}$ encontrava-se reduzido, adotando-se para isto um coeficiente de extinção molar $\mathrm{DE}_{550}=21,0 \times 10^{3} \mathrm{M}^{-1} \mathrm{~cm}^{-1}{ }^{17}$.
Os resultados finais foram expressos, após o agrupamento dos respectivos pontos para cada tempo de coleta de cada paciente. Sendo assim, foi confeccionada uma curva que representava a média destes pontos. Desta curva foi calculada a produção de radical superóxido em nmoles/min, que representou a média do grupo $\mathrm{AI}$, que foi confrontada com os valores dos grupos controles ESP e CT, obtidos de forma semelhante.

$\mathrm{O}$ cálculo final foi dado pela fórmula: Cálculo $\left[\mathrm{O}_{2}{ }^{-}\right.$ ] $=\mathrm{K} \times \mathrm{DO} \times \mathrm{V}$. Onde: $\mathbf{K}$ : constante calculada para o volume final em cada poço. Nesse estudo, $\mathrm{K}=205,49$; DO: densidade ótica; e $\mathbf{V}$ : volume da amostra $(600 \mathrm{ml})$.

Os resultados dos diferentes grupos foram expressos por seus valores absolutos e respectivas médias e desvios padrões. Para verificação da diferença das médias dentro dos grupos foram utilizados o teste " $\mathrm{t}$ " de Student para amostras pareadas e não pareadas. Para a análise da diferença das médias entre os grupos foi utilizada a análise de variância (ANOVA), e quando a ANOVA revelou diferença significativa, foi utilizado o teste de Tukey. A significância estatística foi considerada, admitindo-se um nível crítico de $5 \% \mathrm{em}$ todos os casos ( $\mathrm{p} £ 0,05)$. Foi utilizado o software Jandel Sigma Stat, versão 2.0.

\section{Resultados}

\section{Índice de aderência}

$\mathrm{O}$ índice de aderência foi expresso em porcentagem (\%). Nos monócitos dos pacientes do grupo AI, a média do IA foi de $69,2 \% \pm 9,7 \%$; nos monócitos dos pacientes do grupo ESP, a média do índice de aderência foi de $66,3 \pm 13,4 \%$ e nos monócitos dos sadios do grupo $\mathrm{CT}$, foi de $67,3 \% \pm 14,5 \%$. Comparando as médias dos IA, através da ANOVA, não se observou diferença entre os grupos $F=0,190 ; p=0,827$. (Figura 1)

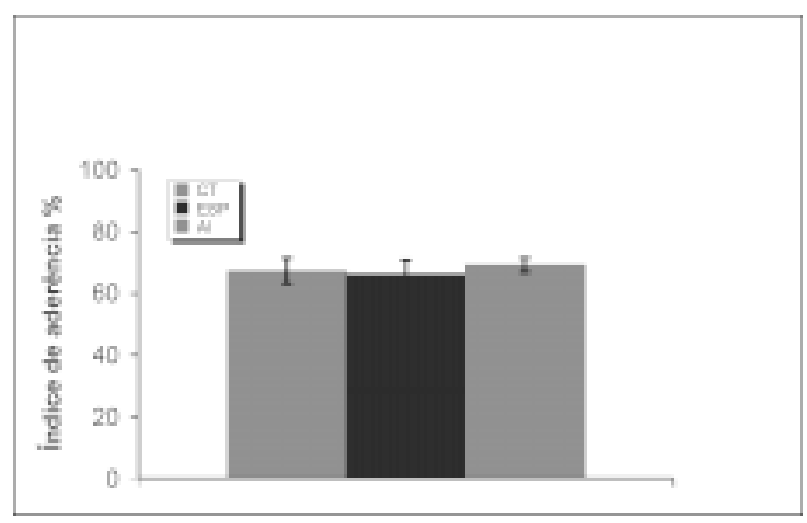

FIGURA 1 - Médias dos IA dos monócitos dos 3 grupos. 


\section{Produção de radical superóxido}

A produção de radical superóxido pelos monócitos foi expressa em nmol $/ \mathrm{min} \times 10^{6} / \mathrm{ml}$. A liberação de $\mathrm{O}_{2}^{-}$ pelos monócitos foi avaliada durante o período de 2 horas.

A produção média de $\mathrm{O}_{2}^{-}$pelos monócitos estimulados com PMA dos indivíduos do grupo CT, aumentou da $1^{\mathrm{a}}$ para $2^{\mathrm{a}}$ hora $(14,5 \pm 4,5$ vs $20,8 \pm 6,2$; $t=-9,049 ; p £ 0,001)$. O mesmo achado foi encontrado nos monócitos estimulados com PMA e tratados com tuftsina (PMA + tuftsina) $(13,5 \pm 5,1 v s 23,2 \pm 8,6$; $\mathrm{t}=$ $-3,849 ; \mathrm{p}=0,003)$. Porém, estes valores não diferem, nem na $1^{\mathrm{a}}$ hora $(14,5 \pm 4,5$ vs $13,5 \pm 5,1 ; \mathrm{t}=0,465 ; \mathrm{p}$ $=0,647)$, nem na $2^{\mathrm{a}}$ hora $(20,8 \pm 6,2$ vs $23,2 \pm 8,6 ; \mathrm{t}=$ $-0,789 ; \mathrm{p}=0,439)$. (Figura 2).

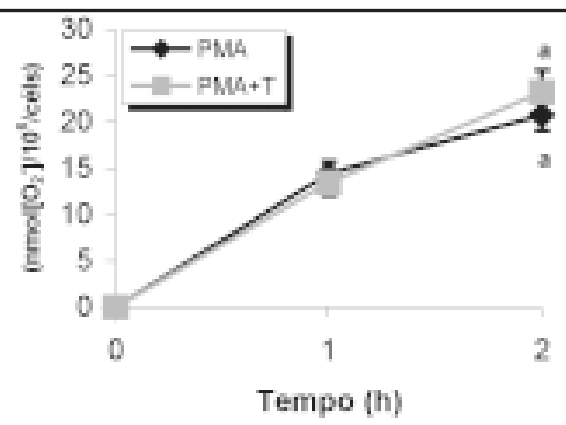

FIGURA 2 - Produção de superóxido (O2-) por monócitos do grupo CT estimulados com PMA e com PMA + Tuftsina. a: diferença de $1^{\mathrm{a}}$ para $2^{\mathrm{a}}$ horas

No grupo ESP, a média da produção de $\mathrm{O}_{2}^{-}$pelos monócitos estimulados com PMA, aumentou da $1^{\mathrm{a}}$ para $2^{\mathrm{a}}$ hora $(4,2 \pm 2,2$ vs $7,1 \pm 2,3 ; \mathrm{t}=-3,436 ; \mathrm{p}=0,009)$. Nos monócitos estimulados com PMA + tuftsina não houve aumento da liberação de $\mathrm{O}_{2}{ }^{-}$da $1^{\mathrm{a}}$ para $2^{\mathrm{a}}$ hora $(3,7 \pm 2,9$ vs $6,7 \pm 3,5 ; \mathrm{t}=-2,282 ; \mathrm{p}=0,052)$. Na comparação da liberação média de radical $\mathrm{O}_{2}^{-}$pelos monócitos estimulados com PMA ou com PMA + tuftsina, observou-se que não houve aumento nem na $1^{\mathrm{a}}$ hora $(4,2 \pm 2,2$ vs $3,7 \pm 2,9 ; \mathrm{t}$ $=0,358 ; \mathrm{p}=0,725)$, nem na $2^{\mathrm{a}}$ hora $(7,1 \pm 2,3$ vs $6,7 \pm$ 3,$5 ; \mathrm{t}=0,290 ; \mathrm{p}=0,776$ ). (Figura 3 ).

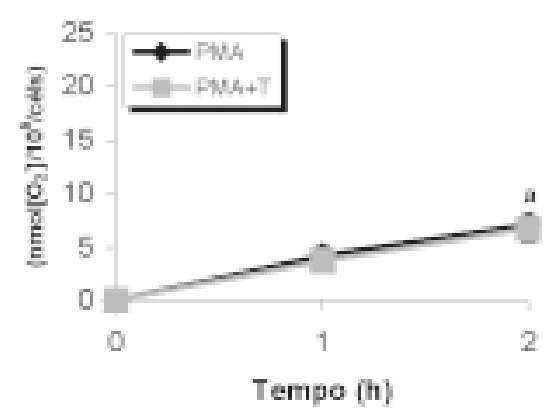

FIGURA 3 - Produção de superóxido (O2-) por monócitos do grupo ESP estimulados com PMA e com PMA + tuftsina. a: diferença de $1^{\mathrm{a}}$ para $2^{\mathrm{a}}$ horas.
A média da produção de $\mathrm{O}_{2}^{-}$dos monócitos dos pacientes do grupo AI, estimulados com PMA, não sofreu aumento entre a $1^{\mathrm{a}}$ e a $2^{\mathrm{a}}$ hora $(14,9 \pm 7,3 v s$ $16,1 \pm 5,6 ; t=-0,541 ; p=0,595)$. Houve aumento da $1^{\mathrm{a}}$ para $2^{\mathrm{a}}$ hora pelos monócitos estimulados com PMA + tuftsina $\left(13,1 \pm 5,6 v_{s} 18,3 \pm 8,5 ; \mathrm{t}=-2,867 ; \mathrm{p}=\right.$ $0,011)$. Porém, comparando a média da produção de $\mathrm{O}_{2}{ }^{-}$nos monócitos estimulados com PMA ou com PMA + tuftsina não se observa diferença, nem na $1^{\mathrm{a}}$ hora $(14,9 \pm 7,3$ vs $13,1 \pm 5,6 ; \mathrm{t}=0,837 ; \mathrm{p}=0,408), \mathrm{nem}$ na $2^{\mathrm{a}}$ hora $(16,1 \pm 5,6$ s $18,3 \pm 8,5 ; \mathrm{t}=-0,917 ; \mathrm{p}=$ 0,366). (Figura 4).

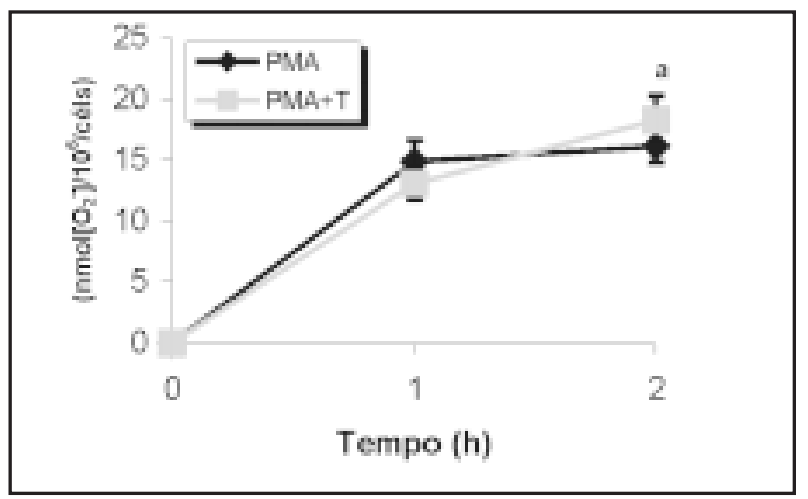

FIGURA 4 - Produção de superóxido $\left(\mathrm{O}_{2}^{-}\right)$por monócitos do grupo AI estimulados com PMA e com PMA + tuftsina. a: diferença de $1^{\mathrm{a}}$ para $2^{\mathrm{a}}$ horas.

A média da produção de radical $\mathrm{O}_{2}^{-}$na $1^{\mathrm{a}}$ hora, pelos monócitos dos pacientes dos grupos AI, ESP e dos indivíduos do grupo CT, estimulados com PMA, mostrou diferença significante (14,9 $\pm 7,3$ vs 4,2 $\pm 2,2$ vs $14,5 \pm 4,5 ; \mathrm{F}=11,7 ; \mathrm{p} £ 0,001)$. Para situar a diferença entre as médias, foi aplicado o teste de Tukey, não revelando diferença entre os grupos $\mathrm{AI}$ e CT (Diferença de média $=0,444 ; \mathrm{q}=0,294 ; \mathrm{p}<0,05$ ). A diferença foi localizada entre os grupos AI e ESP (Diferença de média $=10,7 ; q=6,4 ; p<0,05$ ) e entre os grupos CT e ESP (Diferença de média $=10,2 ; \mathrm{q}=$ $5,7 ; \mathrm{p}<0,05)$. Em relação à média da produção de $\mathrm{O}_{2}$ na $2^{\mathrm{a}}$ hora nos grupos AI, ESP e CT, também ocorreu diferença entre os grupos $(16,1 \pm 5,6 v s 7,1 \pm 2,3$ vs $20,8 \pm 6,2$ vs; $F=17,3 ; p £ 0,001)$. O teste de Tukey não mostrou também diferença entre os grupos AI e CT (Diferença de média $=4,7 ; \mathrm{q}=3,3 ; \mathrm{p}<0,05$ ), situando as diferenças entre os grupos AI e ESP (Diferença de média $=9,0 ; q=5,8 ; p<0,05$ ) e os grupos CT e ESP (Diferença de média $=13,7 ; \mathrm{q}=8,2 ; \mathrm{p}<$ 0,05). (Figura 5). 


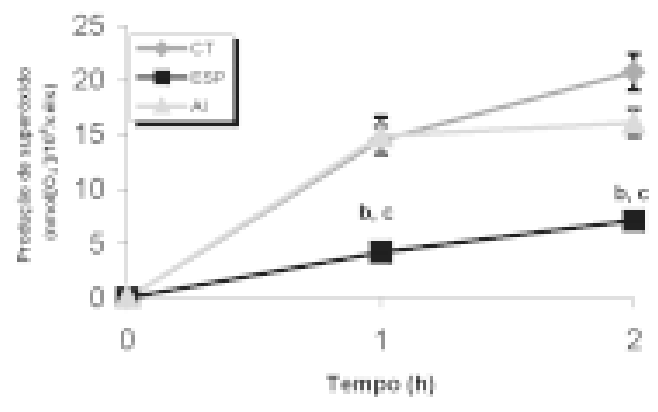

FIGURA 5 - Produção de superóxido $\left(\mathrm{O}_{2}^{-}\right)$por monócitos dos 3 grupos estimulados com PMA.

b: diferença entre ESP e CT; c: entre ESP e AI.

Quando os monócitos foram estimulados com PMA + Tuftsina, a média da produção do radical $\mathrm{O}_{2}^{-}$ na $1^{\text {a }}$ hora nos grupos AI, ESP e CT, mostrou diferença $(13,1 \pm 5,6$ vs $3,7 \pm 2,9$ vs $13,5 \pm 5,1 ; \mathrm{F}=12,4 ; \mathrm{p} £$ $0,001)$. A diferença entre as médias foi localizada pelo teste de Tukey, que, novamente, não revelou diferença entre os grupos AI e CT (Diferença de média $=0,4 ; \mathrm{q}$ $=0,35 ; \mathbf{p}<0,05)$, mas mostrou diferença entre os grupos AI e ESP (Diferença de média $=9,3 ; \mathrm{q}=6,4$; $\mathrm{p}<0,05$ ) e entre os grupos CT e ESP (Diferença de média $=9,8 ; q=6,2 ; p<0,05)$. Em relação a média da produção de $\mathrm{O}_{2}^{-}$na segunda hora, também ocorreu diferença $(18,3 \pm 8,5$ vs $6,7 \pm 3,5$ vs $23,2 \pm 8,6 ; \mathrm{F}=$ $12,0 ; \mathrm{p} £ 0,001)$. O teste de Tukey não mostrou diferença entre os grupos AI e CT (Diferença de média = 4,9; $\mathrm{q}=2,4 ; \mathrm{p}<0,05)$, situando as diferenças, novamente, entre os grupos AI e ESP (Diferença de média = 11,6; $\mathrm{q}=5,1 ; \mathrm{p}<0,05$ ) e os grupos CT e ESP (Diferença de média $=16,5 ; q=6,8 ; p<0,05$ ). (Figura 6).

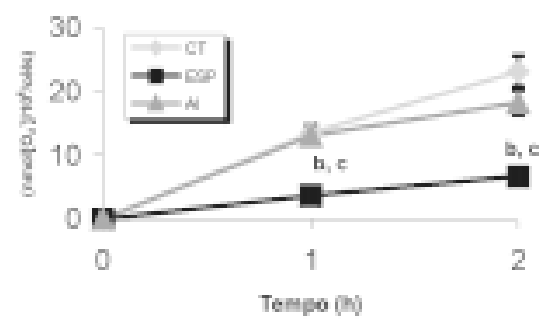

FIGURA 6 - Produção de superóxido $\left(\mathrm{O}_{2}^{-}\right)$por monócitos dos 3 grupos estimulados com PMA + tufsina.

b: diferença entre ESP e CT; c: entre ESP e AI.

\section{Discussão}

A esquistossomose associa-se à pobreza, que obriga o uso de águas contaminadas para o exercício da agricultura, trabalho doméstico e lazer. A região Nordeste é a que apresenta a maior prevalência do Brasil, sendo que mais de $90 \%$ dos pacientes infectados apresentam a forma leve e moderada, e cerca de $4 \%$ a $10 \%$ a forma grave, hepatoesplênica ${ }^{1}$.

Até 1989 , a conduta em crianças portadoras de hipertensão porta esquistossomótica cirúrgica, no Nordeste do Brasil, consistia, de forma similar aos adultos, em esplenectomia associada à sutura obliterante das varizes de esôfago. A morbidade, em termos de recidiva hemorrágica, era semelhante a dos adultos, entretanto, a mortalidade era maior em virtude da sepse fulminante pós-esplenectomia ${ }^{20}$. O Serviço de Cirurgia Geral da Criança do HC da UFPE instituiu, a partir de 1990, de forma inédita, nesses pacientes, o protocolo de esplenectomia, ligadura da veia gástrica esquerda $\mathrm{e}$ auto-implante de tecido esplênico no omento maior.

Com relação à idade entre os grupos estudados, houve diferenças $(F=66 ; p £ 0,001)$. Estas localizaramse entre os grupos ESP e CT $(q=15,3 ; p £ 0,05)$ e ESP e $\operatorname{AI}(q=13,7 ; p £ 0,05)$. Porém, não há referência, na literatura, da interferência da idade no índice de aderência e/ou na geração de radical superóxido nos monócitos. Por isso, essa variável de controle não deve ser considerada como um fator que tenha interfirido nos resultados.

Nos pacientes incluídos não foram observados positividade antigênica para hepatite B. Porém, a pesquisa é importante, pois é conhecida a associação da EHE e a hepatite pelo vírus $\mathrm{B}^{21}$.

Os resultados cirúrgicos estão relacionados à reserva de função hepática desses pacientes ${ }^{4}$. A baixa morbidade e a ausência de óbitos pode ser resultado de cirugia realizada em um grupo de pacientes com boa reserva funcional hepática, já que 12 pacientes foram enquadrados como Child A, os outros como Child B.

É conhecida que a iniciação da resposta imune depende da presença de tecido esplênico. Muito embora o auto-implante de tecido esplênico esteja sendo realizado para restaurar a função imune normal do baço, até agora não se sabe se a esplenose resultante é capaz de iniciar uma reação primária humoral aos antígenos TI- $2^{22}$.

A incidência nula de SFPE sugere que a esplenose mantém algumas das funções de defesa originais do baço ${ }^{8}$. É provável, também, que a antibioticoprofilaxia intensa iniciada na indução anestésica e mantida por sete a dez dias; assim como a administração de 
Penicilina $\mathrm{G}$ benzantina a cada três semanas, no pósoperatório tardio até, pelo menos 14 anos de idade, tenha contribuído.

A ligadura da veia gástrica esquerda bloqueia o desvio entre o sistema venoso ázigo e o portal, diminuindo a pressão nas varizes do terço inferior do esôfago e do fundo gástrico ${ }^{23}$.

Depois que ficou provado que pacientes esplenectomizados $^{9-11}$ e asplênicos apresentavam maior susceptibilidade às infecções por várias bactérias, o auto-implante de tecido esplênico realizado com o objetivo de manter função esplênica residual estimulou um número crescente de pesquisas sobre tal procedimento ${ }^{5}$. Este reveste-se de maior importância na criança, na qual a incidência de SFPE é maior do que no adulto. Por outro lado, a aparente regeneração do tecido esplênico autoimplantado parece ser melhor nos organismos jovens do que nos velhos ${ }^{24}$. Este fato é corroborado em modelo animal $^{25}$.

No Brasil, vários autores têm dado apoio à importância da função do auto-implante e do remanescente esplênico após esplenectomia subtotal, demonstrando os efeitos benéficos na manutenção da função imunológica esplênica ${ }^{5,26,27}$. Do ponto de vista clínico, a esplenose pode ter contribuído, no presente estudo, para não ocorrência de nenhum caso de SFPE.

A ligadura dos vasos gástricos curtos é realizada no último tempo da esplenectomia, a fim de manter a irrigação, por maior tempo possível, do pólo superior do baço, que é usado para o auto-implante. Assim foi conduzida a cirurgia em todos os casos do presente estudo. Esse conceito tem tido o suporte de outros estudos, quando é mantido tecido esplênico residual através da esplenectomia parcial. Nesses experimentos, ocorre, também, a preservação da função fagocitária do pólo superior do órgão ${ }^{26,27}$.

Apesar de ser evidente o beneficio do auto-implante de tecido esplênico ${ }^{5,7,9,22,27-34}$, o assunto ainda é controverso e permanece sob investigação ${ }^{8,35}$.

Além do grande fluxo de sangue, $4 \%$ do volume sanguíneo circulante por minuto, o baço representa um importante ponto de encontro entre a informação antigênica tranportada pelo sangue e o sistema imune. A baixa velocidade de fluxo na polpa vermelha leva a um contato íntimo e prolongado entre os antígenos e os fagócitos ${ }^{36,37}$.

Os macrófagos representam a primeira linha de defesa contra antígenos. Estas células fagocitam as bactérias, liberam mediadores bioativos e citocinas como TNF-a, IL-1, IL-6, IL-8 e outras ${ }^{38}$.

A fagocitose desenvolvida pelas células macrofágicas constitui o início do conjunto das atividades de toda a resposta imunológica, que é constituída por várias etapas: aderência ao substrato, quimiotaxia, ingestão de células e partículas inertes e produção de superóxido ${ }^{19}$.

Tem sido demonstrado que dentro de 30 minutos de aderência monocítica, às bactérias e seus produtos, há iniciação de um conjunto de eventos regulatórios, definido pela rápida mudança nos níveis de RNA-m de muitos proto-oncogenes e de mediadores da inflamação, como a interleucina -1 (IL-1) e o TNF-a ${ }^{39}$. Os macrófagos alveolares não aderentes são incapazes de produzir quantidades suficientes de TNF-a em resposta ao LPS, e, ao contrário, a secreção de TNF-a é aumentada pela aderência ${ }^{40}$. Assim sendo, a aderência do fagócito é crucial para a migração e interação com outras células e componentes da matriz extracelular no sítio inflamatório. Isto é evidenciado pelo fato de que células aderidas apresentam maior capacidade fagocitária e menor complexidade de grânulos ${ }^{41}$.

A separação dos monócitos é feita em função do gradiente de densidade com Histopaque. O plástico, o vidro, o nylon, o microexsudato ou a fibronectina são materiais que asseguram a aderência destas células ${ }^{42}$. Esta capacidade de aderência constitui uma função celular essencial que regula múltiplos fenômenos como ligação, migração, diferenciação e morte celular ${ }^{12,19,42}$.

Os valores de índice de aderência entre os monócitos de adultos normais têm sido relatados entre 55\% e $90 \%{ }^{43}$. No presente estudo, três pacientes do grupo AI, um paciente do grupo ESP e um indivíduo do grupo $\mathrm{CT}$, apresentaram índices de aderência menores do que estes valores. É provável que fatores individuais estejam envolvidos e sejam responsáveis por estes resultados, não podendo ser atribuída esta baixa aderência, portanto, ao procedimento da esplenectomia. Não foi encontrado IA maior do que $90 \%$.

A aderência monocitária pode ser fortemente alterada frente a qualquer estímulo que, ao ativar os macrófagos, induza à liberação de citocinas. Essa propriedade pode avaliar o estado de ativação dos

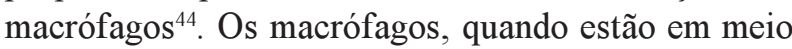
de cultura RPMI, rico em nutrientes e fatores de crescimento celular, apresentam sua aderência normal, constatada através do índice de aderência ao plástico ${ }^{19}$.

Tem sido demonstrado que a aderência dos macrófagos de ratos esplenectomizados é diminuída, contudo, o auto-transplante de tecido esplênico restaura esta atividade funcional ${ }^{45}$. Entretanto, pela análise das médias dos IA dos monócitos dos pacientes dos grupos ESP e AI, encontrando-se valores semelhantes da média do IA dos indivíduos sádios, é possível inferir que a esplenectomia, seguida ou não de auto-implante de 
tecido esplênico, não interfere nesta propriedade das células monocíticas humanas.

O objetivo da ER é gerar reativos microbicidas derivados do oxigênio que podem ser usados pelos fagócitos para a destruição de microrganismos. Este é um mecanismo característico destas células que acompanha a fagocitose. $\mathrm{O}$ interesse foi centrado no ânion superóxido por que a geração deste radical reflete a iniciação da cadeia respiratória ${ }^{46}$.

A redução de citocromo $\mathrm{C}$ por alguns tipos de macrófagos de camundongo, ou monócitos do homem, é linear em 60-90 minutos, e a liberação do ânion superóxido depende da densidade de células em cultura, do tipo de célula, do tipo de estímulo e de outras condições empregadas ${ }^{12}$.

Pela análise da geração média de $\mathrm{O}_{2}$ - dos monócitos dos indivíduos sadios (CT) é possível ter uma idéia da cinética normal da liberação deste radical após estímulo com PMA ou com PMA + tuftsina, donde se observa um aumento na produção de superóxido relacionado ao tempo de estímulo das células ao PMA. Estes achados estão de acordo com a produção de $\mathrm{O}_{2}^{-}$em ratos Wistar normais, estimulados com PMA, observandose elevação crescente dos níveis de produção até as seis primeiras horas, havendo redução a $50 \%$ do valor máximo após $24 \mathrm{~h}$. A partir daí, os valores são quase imensuráveis ${ }^{48}$. Porém, o adicionamento da tuftsina sintética não parece aumentar a média de liberação nas duas horas, não alterando, portanto, a cinética da geração de $\mathrm{O}_{2}^{-}$pelos monócitos do grupo $\mathrm{CT}$.

Os monócitos do grupo ESP, estimulados com PMA, demonstraram aumento da liberação de $\mathrm{O}_{2}^{-}$da primeira para segunda hora, portanto relacionada ao tempo de exposição ao PMA. Aumento não foi observado após estímulo com PMA + tuftsina. É possível inferir que os monócitos do pacientes esplenectomizados são capazes de gerar ânion superóxido em quantidade crescentes, parecendo simular a cinética de geração dos indivíduos sadios. Porém, estas quantidades são muito pequenas, o que, naturalmente, demonstram uma atividade microbicida menor. Não há diferença, como observado no grupo CT, entre a quantidade média de radical superóxido pelos monócitos estimulados com PMA ou com PMA + tuftsina. Fica patente, também, que a administração de tuftsina não altera a cinética da liberação de $\mathrm{O}_{2}$. Tem sido demonstrado que nos pacientes esplenectomizados ocorre diminuição dos níveis de tuftsina ${ }^{32}$. Portanto, seria esperado que os monócitos destes pacientes respondessem, com a administração deste peptídeo, liberando maiores quantidades de $\mathrm{O}_{2}^{-}$.
Nos pacientes do grupo AI não houve aumento na geração média de $\mathrm{O}_{2}^{-}$pelos monócitos estimulados com PMA, relacionado ao tempo. Porém, a liberação de $\mathrm{O}_{2}^{-}$, por estas células, já é satisfatória na $1^{\mathrm{a}}$ hora, já que é semelhante à dos indivíduos controles normais. Aumento foi observado nos monócitos estimulados com PMA + tuftsina. Porém, quando se compara a geração média de $\mathrm{O}_{2}{ }^{-}$pelos monócitos estimulados com PMA ou com PMA + tuftsina não se observa diferença, o que demonstra, também, que a tuftsina não altera a cinética dos monócitos neste grupo de pacientes. Os níveis séricos de tuftsina em crianças esquistossomóticas esplenectomizadas seguidas de auto-implante de tecido esplênico no omento maior são similares aos das crianças sadias, o que demonstra que este procedimento é um meio eficiente de manter as concentrações deste peptídeo ${ }^{9}$. Nos pacientes que desenvolvem algum grau de esplenose após lesões traumáticas, as concentrações da tuftsina também situam-se em níveis normais $^{32}$.

Quando foram comparadas as médias da produção de $\mathrm{O}_{2}^{-}$pelos monócitos estimulados com PMA, entre os grupos AI, ESP e CT, observa-se que os monócitos dos pacientes submetidos ao auto-implante de tecido esplênico liberam $\mathrm{O}_{2}^{-}$em quantidades semelhantes aos monócitos do grupo controle e que esta produção foi maior do que a produção no grupo de pacientes esplenectomizados. Estes achados podem demonstrar que o auto-implante de tecido esplênico contribui de forma sinérgica ou isolada para que o monócito tenha uma atividade microbicida normal, pela manutenção das reações imunes que continuam se processando na esplenose.

Os monócitos dos pacientes esplenectomizados parecem ter a capacidade de se aderir à superfície da bactéria, mas a resposta imune não é completada pela falta do baço. Estes dados podem suportar a hipótese de que, por um lado, a produção de $\mathrm{O}_{2}$ - deficiente nos pacientes esplenectomizados seria um dos fatores implicados na resposta deficitária do hospedeiro às infecções, permitindo a instalação da SFPE, e por outro, que o auto-implante de tecido esplênico restaura em parte a capacidade microbicida dos monócitos. O benefício deste procedimento, portanto, seria manter a integração do sistema fagocítico mononuclear (SFM), pivô no mecanismo primário de defesa do hospedeiro, através de seus sistemas microbicidas. Pode-se sugerir ainda que a liberação de superóxido pode representar um marcador do processo patológico que ocorre durante a SFPE. Os mesmos achados foram encontrados, entre os grupos, comparando as médias da geração de superóxido pelos monócitos estimulados com PMA + tuftsina ${ }^{47}$. 
Os achados estão, de certa forma, em concordância com a avaliação similar feita em pacientes esplenectomizados por trauma esplênico. Nesse grupo de pacientes foi observado, ainda, diminuição da atividade intracelular da mieloperoxidase ${ }^{48}$. Igualmente, tem o respaldo de estudo da função fagocítica dos macrófagos em ratos que diminui após esplenectomia parcial. Entretanto, o remanescente esplênico tem a capacidade de ser estimulado quando exposto a um estímulo antigênico ${ }^{49}$.

Mizukami e col..$^{50}$ mostraram que a exposição de ratos a polissacarídios da cápsula de pneumococos resulta em aumento na liberação de superóxido pelas células de Kupffer dentro dos sinusóides e dano das células endoteliais nos sinusóides. Estas alterações foram atenuadas pelo tratamento com superóxido dismutase ou pela esplenectomia, demonstrando que este procedimento reduz a produção de $\mathrm{O}_{2}^{-}$, tendo efeito protetor sobre a injúria hepática induzida pelo polissacarídio ${ }^{50}$.

McCarthy e col. ${ }^{11}$ comparando grupos de ratos adultos, controles, esplenectomizados e outro grupo submetido a operações simuladas, avaliaram defeitos na função dos macrófagos peritoneais no período precoce da esplenectomia e verificaram que a produção de superóxido pelos macrófagos não foi prejudicada em nenhum momento. Porém, foram encontradas taxas de mortalidade em 24h maior no grupo esplenectomizado além da redução da liberação de óxido nítrico (um outro produto microbicida), leucopenia e redução da atividade microbicida intracelular em 24h. Este último defeito na função dos macrófagos ocorreu apesar da liberação de quantidades similares de superóxido. Os autores afirmaram que é inteiramente possível que outro mecanismo antibacteriano seja responsável por estes achados. Em contraste, a atividade microbicida extracelular não foi prejudicada após a esplenetomia, e este achado é correlacionado com o aumento na produção de $\mathrm{O}_{2}^{-}$após a esplenectomia, uma resposta antecipada dos macrófagos na presença de certos micróbios ${ }^{11}$.

A idéia geral foi dar maior consistência à hipótese de que o auto-implante de tecido esplênico pode influir de forma positiva na atividade fagocítica, na imunidade celular e humoral destes pacientes, o que pode ser capaz de reduzir a incidência da sepse fulminante pósesplenectomia. Outra alternativa com fundamentação similar é a esplenectomia parcial nesses pacientes, cujos resultados satisfatórios têm sido confirmados em adultos $^{49,51}$.

Até agora não existem experimentos que avaliem diretamente a função do baço. A forma de avaliação ideal da capacidade fagocítica das células macrofágicas esplênicas poderia ser estudar, tanto funcional como histologicamente, os macrófagos dos fragmentos esplê- nicos auto-implantados neste grupo de pacientes. Esse estudo, obviamente, não responde a questão crítica se o auto-implante de tecido esplênico fornece tecido esplênico capaz de prevenir a SFPE. Porém, é possível que traga subsídio para pesquisas futuras nesta área.

\section{Conclusões}

1 - O índice de aderência dos monócitos dos grupos CT, ESP e AI foi semelhante.

2 - A atividade microbicida de monócitos estimulados com PMA e com PMA + tuftsina, avaliada pela liberação de $\mathrm{O}_{2}^{-}$, em pacientes esplenectomizados com auto-implante de tecido esplênico foi semelhante a liberação em indivíduos normais na primeira e segunda hora e esta liberação foi maior em relação aos pacientes esplenectomizados.

3 - O tratamento dos monócitos com tufsina não alterou a cinética da geração de superóxido nos três grupos.

\section{Referências}

1. Katz N, Peixoto SV. Análise crítica da estimativa do número de portadores de esquistossomose mansoni no Brasil. Rev Soc Bras Med Trop 2000; 33:303-8.

2. Brandt CT, Frei Caneca OA, Tavares DJS, Santos de Azevedo FA, Agra Jr VG, Cordeiro F. Hipertensão portal esquistossomótica na criança: avaliação crítica. An Fac Med Uni Fed Pernam 1995; 40:106-10.

3. Brandt CT, Frei Caneca OA, Tavares DJS, Ávila JR. Surgical hepatosplenic mansonic schistosomiasis in children: a Dopller Duplex study of the portal vein and hepatic artery. Trans $r$ Soc Trop Med Hyg 1995; 89:70-1.

4. Sakai P, Maluf F, Gurgel J, Ishioka S. Late results of endoscopic sclerotherapy of bleeding esophageal varices in patients with hepatic cirrhosis and schistosomiasis. Arq Bras Cir Dig 1995; $10: 17-21$

5. Brandt CT, Oliveira BS, Nogueira JBM, Neves JR, Lopes TL. Esquistossomose hepatoesplênica em crianças: avaliação morfológica e funcional após esplenectomia e auto-implante esplênico. Rev Col Bras Cir 1998; 25: 399-402.

6. Singer DB. Postsplenectomy sepsis. Perspectives in Paediatric Pathology 1976; 1:285-311.

7. Vega A, Howell C, Krasna I, Campos J, Heyamn S, Ziegler M, Koop E. Splenic autotransplantation: optimal functional factors. J Ped Surg 1981; 16:898-904.

8. Lüdtke FE, Mack SC, Schuff-Werner P, Noth E. Splenic function after splenectomy for trauma. Role of autotransplantation and splenosis. Acta Chi Scand 1989; 155:533-9.

9. Brandt CT, Maciel DT, Caneca OAF, Castro CMMB, Araújo LB. Autotransplant of spleen tissue in children with schistosomiasis: evaluation of splenic function after splenosis. Mem Inst Oswaldo Cruz 2001; 96: 117-22

10. Hathaway JM, Harley RA, Sally SELF, Schieffman G, Virella $\mathrm{G}$. Immunological function in post-traumatic splenosis. Clin Immunol Immunopathol 1995; 74:143-50.

11. Mccarthy JE, Remond PH, Duggan SM, Watson RW, Condron CM, Donnel JR, Bouchier-Hayes DJ. Characterization of the defects in murine macrophage function in the early postsplenectomy period. J Immunol 1995; 155:387-96. 
12. Segura JJ, Jiménez-Rubio A. Effect of eugenol on macrophage adhesion in vitro to plastic surfaces. J Endod 1998; 24 . 229-31.

13. Babior BM. Superoxide: a two-edged sword. Braz J Med 1997; 30: 141-55.

14. Malorni W, Testa U, Rainaldi G, Tritarelli E, Peschle C. Oxidative stress to a rapid alteration of transferrin receptor intravesicular trafficking. Exp Cell Res 1998; 241:102-16.

15. Glass GA, Delisle DM, Detogni P, Gabig TG, Magee BH. The respiratory burst oxidase of human neutrophils. J Biol Chem 1986; 264: 13247-51.

16. Roos D, Winterbourn CC. Lethal Weapons. Science 2002; 296: 669-71

17. Johnston Jr RB. Measurement of O2- secreted by monocytes and macrophages. Methods in enzimology 1984; 105:365-9.

18. Cohn ZA. The activation of mononuclear phagocytes: fact, fancy, and future. Journal of Immunology 1978; 121: 813-6.

19. de la Fuente M, Del Rio M, Ferrandez MD, Hernanz A. Modulation of phagocytic function in murine peritoneal macrophages by bombesin, gastrin-releasing peptide and neuromedin. C Immunol 1991; 73: 205-11.

20. Kelner S. Critical evaluation of surgical treatment of schistosomiasis portal hypertension. Mem Inst Oswaldo Cruz 1992; 87: 357-68.

21. Meira MRL, Figuerêdo-Silva J, Silveira MJC, Kelner S, Montenegro LT. Surgical hepatoesplênic schistosomiasis and hepatite B: a serological, histological and immunohistochemical study of 30 cases. An Fac Med Uni Fed Pernam 1999; 44: 77-81.

22. Leemans R, Beekhuis H, Timens W, Hauw The T, Klasen HJ. Fc-receptor function after splenic autotransplantation. Bri j Surg 1996; 83:543-6

23. Lacerda CM, Freira W, Melo PSV, Carvalho GL, Kirzner CF. Esplenectomia e ligadura da veia gástrica esquerda na esquistossomose mansônica: efeito sobre a pressão das varizes de esôfago aferida por técnica não invasiva. Rev Col Bras Cir 1998; 26: 15-9

24. Jugenburg M, Haddock G, Freedman MH, Ford-Jones L, Ein SH. The morbidity ands mortality of pediatric splenectomy does prophylaxis make a diference? J Ped Surg 1999; 34: 1064-7.

25. Corazza GR, Zoli G, Massai G, Mile P, Beltrandi F, Gasbarrin G Changes in perifheral blood lymphocytes and immune complexes in splenectomized patients: lach of correlation with residual splenic with residual splenic function. J Clin Lab Immunol 1990, 31:33-8.

26. Petroianu A, Antunes LJ. Immune profiles in hepatosplenic schistosomiasis mansoni after surgical treatments. J Int Med Res 1998; 26:43-9.

27. Resende V, Petroianu A. Estudo funcional tardio do autoimplante esplênico após trauma complexo do baço humano. Rev Col Bras Cir 2001; 28:165-70.

28. Coney DR, Dearth JC, Swanson SE, Dewanjee MK, Telander RC. Relative merits of partial splenectomy, splenic reimplantation and immunization in preventing postsplenectomy infection. Surgery 1979; 86: 561-9.

29. Traub A, Giebink GS, Smith C, Kuni C, Brekke ML, Edlund D, Perry J. Splenic reticuloendothelial function after splenectomy, spleen repair and spleen auto-transplantation. New Engl J Med 1987; 317:1159-64.

30. Shokouh-Amiri MH, Kharazami A, Rahimi-Saber S, Hansen CP, Jensen SL. Phagocyte function after splenic autotransplantation. Arch Surg 1990; 125:595-7.

31. Bergmann L, Bttcher W, Seufert RM, Mitrou PS. Quantitative and functional restorations and alterations of perifheral lymphocyts in patients with autologous spleen implantation. Arch Orthop Trauma Surg 1990; 109: 102-5.

32. Zoli G. Splenic autotransplantation after splenectomy: tuftisin activity correlates with residual splenic function. Br J Surg 1994; 81: 716-8

33. Brandt CT, Araújo LB, Castro CMMB. Autotranspalantation of spleen tissue in children with mansonic schistosomiasis who underwent splenectomy: evaluation of splenic residual functions. Acta Cir Bras 1998; 13: 212-6.

34. Leemans R, Harms G, Rijkers GT, Timens W. Spleen autotransplantation provides restoration of functional splenic lymphoid compartments and improves the humoral immune response to pneumococcal polysaccharide vaccine. Clin Exp Immunol 1999; 117: 596-604.

35. Winkelstein JA, Lambert GH. Pneumococical serum opsonizing activity in splenectomized children. J Pediatric 1975; $87: 430-3$.

36. Timens W, Leemans R. Splenic autotransplantation and the immune system. Ann Surg 1991; 215: 256-60.

37. Kays MA, Stolar CJH. The spleen and splenectomy: implication for the pediatric population. In Fkalsrud EW, Krumel TM. Infectious and immunologic disorders in pediatric surgery. Philaldelphia: W. B Saunders Company; 1993. p. 91-100.

38. Malangoni M, Evers BM, Peyton JC, Wellhausen SR. Reticuloendothelial clearance and splenic mononuclear cell populations after resection and autotransplantation. Am J Surg 1988; 155:302.

39. Zareie M, Singh PK, Irvine EJ, Sherman PM, Mckay DM, Perdue MH. Monocyte/macrophage activation by normal bacteria and bacterial products. Implications for altered epithelial function in Crohn's disease. Am J Pathol 2001; 158: $1101-9$

40. Kelley JL, Rozek MM, Suenram CA, Schwartz CJ. Activation of human blood monocytes by adherence to tissue culture plastic surfaces. Exp Mol Pathol 1987; 46: 266-78.

41. Skuld CM, Barck C, Lundahl J, Johansson A. Different functional and morphological characteristics in a nonadherent subpopulation of human macrophages recovered by bronchoalveolar lavage. Eur Resp J 1995; 8:1719-24.

42. Noga SJ, Normanm SJ, Winer RS. Methods in laboratory investigation -isolation of guinea pig monocyte and Kurloff cells: characterization of monocyte subsets by morphology, cytochemistry, and adherence. Labor Invest 1984; 51: 244-52.

43. Cooper MJ, Williamson RCN. Splenectomy: indications, hazards and alternatives. Br J Surg 1984; 71:173-180.

44. Sporn SA, Eierman DF, Johnson CE, Morris J, Martin G, Ladner $\mathrm{M}$, Haskill S. Monocyte adherence results in selective induction of novel genes sharing homology with mediators of inflammation and tissue repair. J Immunol 1990; 144: 4434-41.

45. Bauer GJ, Arbabi S, Garcia IA, Dehingh I, Rosengart M, Maier R. Adherence regulates macrophage signal transduction and primes tumor necrosis factor production. Shock 2000; 14: 435-40.

46. Aker M, Ziran A, Abrahamov A, Horowitz M, Matzner Y. Abnormal neutrophil chemotaxis in Gaucher disease. Br J Haematol 1993; 83:187-91.

47. de Castro CMMB, Manhães De Castro R, Medeiros A, Santos AQ, Silva WTFE, Lima Filho JL. Effect of stress on the production of $\mathrm{O}_{2}$ in alveolar macrophages. J Neuroimmunol 2000; 108: 68-72.

48. Wysochi H, Wierusz-Wysocka B, Karon H, Dotka J, Wykretowicz A, Szczepanik A, Klimas R. Polymorphonuclear neutrphils function in splenectomized patients. Clin Exp Immunol 1989; 75:392-5. 
49. Müftüoglu TMA, Köksal N, Özkutlu D. Evaluation of phagocytic function of macrophages in rats after partial splenectomy. Am Coll Surg 2000; 191: 668-71

50. Mizukami T, Yokoyama H, Okamura Y, Ohgo H, Fukuda M, Kamegaya Y, Kato S, Ishi H. Splenectomy attenuates superoxide anion release into the hepatic sinusoid after lipopolysaccharide challenge. J Hepatol 1999; 31: 235-41.

51. Petroianu A, Silva RG, Simal CJR, Carvalho DG, Silva RAP Late postoperative follow-up of patients submitted to subtotal splenectomy. Am Surgeon 1997; 63: 735-40.

Brandt CT, Leite CRC, Manhaes-de-Castro R, Brandt Filho C, Castro CMMB. Monocyte adherence and microbe kill activity in patients with surgical schistosomisis mansoni. Acta Cir Bras [serial online] 2003 Mar-Apr;18(2). Available from URL: http://www.scielo.br/acb.

ABSTRACT - Surgical treatment in children suffering from schistosomissis mansoni includes splenectomy; ligature of left gastric vein and auto-implantation of spleen morsels into the greater omentum. The efficacy of this procedure may be responsible for the disappearance of overwhelming post-splenectomy infection (OPSI), in this kind of patients. This condition may be assigned to the lowering of $\operatorname{IgM}$, circulating lymphocytes, properdin and to the absence of tutfsin, which may lead to the deficiency of mono-macrophage activity cells, responsible for bacteria adherence, phagocytosis and its destruction. Purpose: To analyze the functional features of the monocytes from young patients, who underwent splenectomy, ligature of left gastric vein and auto-implantation of morsels of spleen tissue on the major omentum. These patients were cared for at - University Hospital «Serviço de Cirurgia Geral da Criança, Hospital das Clínicas, UFPE», from 1991 to 2000. Methods: It was analyzed the rates of monocytes in vitro adherence to the solid surface and the generation of $\mathrm{O}_{2}^{-}$radical by the monocytes, stimulated with acetate miristate of phorbol (PMA) and PMA + tuftsin, in 3 groups. The $1^{\text {st }}$ group (AI), constituted by 18 patients with schistosomiasis mansoni in the hepatosplenic form and treated with splenectomy, ligature of left gastric vein and autoimplantation of fragments of spleen tissue on the major omentum; the $2^{\text {nd }}$ group (ESP), formed by 09 patients with schistosomiasis mansoni in the hepatosplenic form who underwent azigo-portal disconnection with splenectomy and the $3^{\text {rd }}$ one (CT) constituted by 12 voluntary teenagers from the same geographic area and with the same socio-economical conditions. Results: There was no significant difference in the monocyte adherence rate among the 3 groups. With regard to the generation of $\mathrm{O}_{2}^{-}$release, the monocytes of patients from AI group had a super oxide generation similar to that of individuals from CT group, and higher than the patients from ESP group. The monocyte treatment with tuftsin did not change the kinetic of the superoxide generation on the 3 groups. Conclusions: The monocytes of the surgical hepatosplenic schistosomiasis mansoni bearers who underwent splenectomy, ligature of the left gastric vein and auto-implantation of spleen tissue into the major omentum showed functional similar features as comparared to normal people from the same social-economical-geographical conditions.

KEY WORDS - Schistosomiasis mansoni. Monocyte. Microbe kill activity.

Conflito de interesse: nenhum

Fonte de financiamento: nenhuma

Correspondência:

Carlos Teixeira Brandt

Av. Boa Viagem, 5090/1201

50011-000 Recife - PE

carlosbrandt@bol.com.br

Data do recebimento: 18/11/2002

Data da revisão: 12/12/2002

Data da aprovação: 05/01/2003 